\title{
Editorial
}

Nephrology

\section{Why Is the Association of Phosphorus and FGF23 with Mortality Stronger in African-American Hemodialysis Patients?}

\author{
Wei Ling Lau Kamyar Kalantar-Zadeh \\ Nephrology, University of California, Irvine, Calif., USA
}

Mortality rates and prevalence of kidney disease are significantly different between African-Americans and Whites in the United States. This holds true in both the non-dialysis-dependent chronic kidney disease and endstage renal disease (ESRD) populations, but the trends are opposite in these distinct patient groups. AfricanAmericans comprise $14 \%$ of the U.S. general population but have an incident rate of ESRD that is 3-fold greater than in non-Hispanic Whites such that $\sim 35 \%$ of the ESRD population are African-American $[1,2]$. Minority groups have a shorter life expectancy in the general population, and this holds true in non-dialysis-dependent chronic kidney disease. Analysis of 2,900 CKD patients from National Health and Nutrition Examination Survey III data showed that African-Americans $<65$ years of age have a $78 \%$ higher mortality than non-Blacks [3]. This risk was attenuated by adjustment for socioeconomic factors, suggesting a major influence of high-risk lifestyle events such as homicide and substance abuse [3].

In stark contrast, African-Americans have a substantially greater survival than Whites in the ESRD population $[4,5]$. This survival advantage is particular prominent among Blacks above age 50, the age range that comprises the bulk of the ESRD population [2]. Analysis of $>124,000$ maintenance hemodialysis (MHD) patients from the national DaVita cohort showed persistence of the survival advantage even after adjustment for demo-

\section{KARGER 125\%}

(๑) 2015 S. Karger AG, Basel

0250-8095/15/0421-0022\$39.50/0

E-Mail karger@karger.com

www.karger.com/ajn graphics, dialysis modality and cause of death [4]. Nutritional status and inflammation play a major part in this mortality difference. Blacks on MHD have higher average lean body mass and prealbumin levels than their White counterparts [6]. The mortality difference between Blacks and Whites is most pronounced at higher levels of Creactive protein and interleukin-6 (IL-6) [5]. Analysis by race suggests that Whites are more susceptible to the effects of inflammation, whereby the highest vs. lowest quartile of IL- 6 corresponds with $4.1 \times$ greater mortality risk in Whites compared to $2.4 \times$ in Blacks [6]. However, adjustment for markers of nutrition and inflammation (after traditional case-mix adjustment) reverses the risk profile and places African-Americans on MHD at a higher mortality risk than Whites [4].

What else could account for these racial disparities in ESRD survival? Aside from ethnic differences in nutrition and inflammatory profiles, factors that have been proposed include cultural differences, genetic differences that impact dialysis response (such as APOL1 gene), and variation in mineral bone disease (MBD) metabolism [1]. With respect to the latter, exposure to activated vitamin $\mathrm{D}$ analogs appears to confer a significant race-modulated survival advantage. Analysis of MBD parameters in $\sim 140,000$ MHD patients (32\% African-American) showed best survival in Blacks who received the highest doses of vitamin $\mathrm{D}$ agonist (reported as paricalcitol 
$>10 \mu \mathrm{g} /$ week) [7]. A relatively higher serum calcium range of $9.5-10.2 \mathrm{mg} / \mathrm{dl}$ correlated with lowest mortality in the African-American group [7]. Average parathyroid hormone levels are higher in African-Americans, which likely drives vitamin D agonist exposure in this group [7]. Interestingly, a separate analysis of $\sim 107,000$ MHD patients confirmed that higher paricalcitol doses shifted the serum calcium range associated with greatest survival to the right across all races (from $9.0-<9.5$ to $9.5-<10.0$ $\mathrm{mg} / \mathrm{dl}$ ) [8]. Animal studies suggest that beneficial effects downstream of vitamin $\mathrm{D}$ receptor activation include restoration of circulating klotho levels, decreased fibroblast growth factor-23 (FGF23) and decreased arterial calcification [9].

In this issue of Am J Nephrol, Scialla et al. [10] correlate MBD parameters with mortality outcomes in 511 incident hemodialysis patients (36\% African-American) from the Choices for Healthy Outcomes in Caring for ESRD study. The cohort is of historical interest; patients were enrolled from Dialysis Clinic, Inc. centers (Nashville, Tenn., USA) between 1995 and 1998, in the era predating widespread use of vitamin D analogs (12\% of the cohort received intravenous calcitriol). Baseline measurements were done from blood samples collected 4-5 months after hemodialysis initiation, and median duration of follow-up was 3.4 years. Data confirm survival advantage in African-American patients, significant at 8 years out, though at this timepoint only $\sim 50$ patients were surviving. Compared to non-African-American counterparts, Black patients had higher average parathyroid hormone and total alkaline phosphatase levels, and lower calcium, phosphorus, FGF23 and 25-hydroxy vitamin D levels. On continuous analyses with adjustment for demographics, comorbidities, hemoglobin and albumin, only phosphorus and FGF23 correlated with increased mortality risk in all-comers. When stratified by race, the mortality-association of phosphorus and FGF23 remained significant only in Blacks. Due to limited sample size, the investigators also performed dichotomous analyses of the data by race. Figure $1 \mathrm{C}$ displays the most striking finding of the study, whereby African-Americans with FGF23 levels $<1,590 \mathrm{pg} / \mathrm{ml}$ had $2 \times$ better survival than African-Americans with FGF23 levels $\geq 1,590 \mathrm{pg} / \mathrm{ml}$ ( $p=0.005$ between African-American groups); the latter group had survival similar to non-Blacks with low FGF23. Non-Blacks with high FGF23 had the poorest survival [10].

The results of this study are somewhat consistent with the racial FGF23 differences reported in the Accelerated Mortality on Renal Replacement study, which reported that African-Americans with FGF23 levels below the population median had a $60 \%$ lower risk of death as compared to Whites in the same category, in a prospective cohort of $\sim 10,000$ incident hemodialysis patients [11]. An elevated FGF23 level is a marker for phosphorus overload, which promotes vascular calcification; FGF23 itself appears to drive ventricular hypertrophy. Further, elevated FGF23 in MHD patients has been linked with metabolic risk factors unrelated to mineral metabolism, including lower body mass index and lipid abnormalities [12]. The authors concede that it is difficult to ascertain if the mortality associations reflect true biological impact of MBD derangements in African-Americans, or if genetic differences such as APOL1 variants are affecting cardiovascular disease pathogenesis [10].

There were significant differences in baseline characteristics of the two racial cohorts, perhaps due to small sample sizes [10]. The average age was 54.6 vs. 59.8 years in the African-American vs. non-African-American groups; thus the non-Black group may have had higher comorbid disease burden. Levels of inflammatory markers C-reactive protein and IL-6 were higher in the nonBlack group; surprisingly, the authors report that adjustment for inflammatory markers on sensitivity analyses did not modify the results. As discussed earlier, prior studies have noted significant inflammation-related mortality risk in ESRD that is more pronounced in Whites [4-6].

In summary, phosphorus and FGF23 relationships with mortality vary by race, suggesting that variances in mineral metabolism contribute to the survival advantage observed in the African-American hemodialysis population.

\section{Disclosure Statement}

The authors declare no conflict of interest.

References

1 Kalantar-Zadeh K, Golan E, Shohat T, Streja E, Norris KC, Kopple JD: Survival disparities within American and Israeli dialysis populations: learning from similarities and distinctions across race and ethnicity. Semin Dial 2010;23:586-594.

2 Kucirka LM, Grams ME, Lessler J, et al: Association of race and age with survival among patients undergoing dialysis. JAMA 2011; 306:620-626

3 Mehrotra R, Kermah D, Fried L, Adler S, Norris $\mathrm{K}$ : Racial differences in mortality among those with CKD. J Am Soc Nephrol 2008;19: 1403-1410. 
4 Streja E, Kovesdy CP, Molnar MZ, et al: Role of nutritional status and inflammation in higher survival of African American and Hispanic hemodialysis patients. Am J Kidney Dis 2011;57:883-893.

5 Crews DC, Sozio SM, Liu Y, Coresh J, Powe NR: Inflammation and the paradox of racial differences in dialysis survival. J Am Soc Nephrol 2011;22:2279-2286.

6 Noori N, Kovesdy CP, Dukkipati R, et al: Racial and ethnic differences in mortality of hemodialysis patients: role of dietary and nutritional status and inflammation. Am J Nephrol 2011;33:157-167.
7 Kalantar-Zadeh K, Miller JE, Kovesdy CP, et al: Impact of race on hyperparathyroidism, mineral disarrays, administered vitamin $\mathrm{D}$ mimetic, and survival in hemodialysis patients. J Bone Miner Res 2010;25:2724-2734.

8 Miller JE, Kovesdy CP, Norris KC, et al: Association of cumulatively low or high serum calcium levels with mortality in long-term hemodialysis patients. Am J Nephrol 2010;32: 403-413.

9 Lau WL, Leaf EM, Hu MC, et al: Vitamin D receptor agonists increase klotho and osteopontin while decreasing aortic calcification in mice with chronic kidney disease fed a high phosphate diet. Kidney Int 2012;82:12611270.
10 Scialla J, Parekh R, Eustace J, et al: Race, mineral homeostasis and mortality in patients with end-stage renal disease on dialysis. Am J Nephrol 2015;42:25-34.

11 Gutiérrez OM, Mannstadt M, Isakova T, et al: Fibroblast growth factor 23 and mortality among patients undergoing hemodialysis. N Engl J Med 2008;359:584-592.

12 Montford JR, Chonchol M, Cheung AK, et al: Low body mass index and dyslipidemia in dialysis patients linked to elevated plasma fibroblast growth factor 23. Am J Nephrol 2013;37: 183-190. 